\title{
Ultrassom Intracoronário e a Tomada de Decisão no Implante de Stents: Revisando Conceitos
}

\author{
Áurea J. Chaves
}

O stents farmacológicos representaram grande avanço no tratamento percutâneo da doença coronária, ao reduzir a reestenose coronária a valores de um dígito. Entretanto, quando esse fenômeno ocorre, são necessárias novas intervenções, com custos relacionados a recursos humanos e materiais, algumas vezes substanciais. Parte dessas reestenoses pode ser evitada, empregando-se as evidências existentes para guiar um implante ótimo dos stents. Nesse sentido, trazemos nesta edição editorial de Gary Mintz, da Cardiovascular Research Foundation (Nova York, Estados Unidos), que revisa a contribuição que o ultrassom intracoronário traz para a otimização do implante dos stents, discutindo em detalhes os achados de Brito et al. na busca de um preditor de reestenose de stent farmacológico. Lembra ainda outras variáveis ultrassonográficas de interesse associadas à reestenose, potencialmente corrigíveis e com frequência negligenciadas pelos intervencionistas, e a necessidade de se interpretar os resultados de estudos relacionados ao tema estritamente dentro de seu contexto.

Trazemos também contribuição original de Campos et al., que relata os resultados clínicos iniciais do primeiro stent de cromo-cobalto fabricado no Brasil, cujos resultados experimentais já foram relatados em edição prévia da Revista Brasileira de Cardiologia Invasiva. Em editorial correspondente, Chamié e Abizaid, do Instituto Dante Pazzanese de Cardiologia (São Paulo, SP), fazem completa revisão das propriedades estruturais de um stent ideal, com tabela que compara as principais características das endopróteses mais utilizadas em nosso meio, e apontam etapas a serem vencidas pelo stent brasileiro. Estudo comparativo com stents de cromo-cobalto já aprovados faz-se necessário, para confirmar sua eficácia e segurança, além de um custo atrativo, para poder torná-lo alternativa competitiva em nossa prática clínica diária.

Outra excelente contribuição, de Abelin et al., apresenta os resultados de grande coorte de pacientes do registro do Instituto de Cardiologia do Rio Grande do Sul/Fundação Universitária de Cardiologia (Porto Alegre, RS), tratados com stents coronários. Robert Bersin, do Seattle Cardiology and Swedish Medical Center (Seattle, Estados Unidos), enaltece os resultados encontrados, mas se surpreende com a ainda pe- quena utilização dos stents farmacológicos no Brasil. Infere os potenciais benefícios que poderiam ser alcançados com a utilização dessas endopróteses em maior escala e sugere que apelo seja feito a órgãos governamentais e indústria, para que encontrem soluções que permitam que maior proporção de pacientes tenha acesso às novas tecnologias.

Da América Latina recebemos artigos originais que tratam dos resultados da intervenção coronária percutânea no infarto agudo do miocárdio. Sousa et al. comparam o desempenho da angioplastia primária ao longo dos anos em mais de 40 mil pacientes do Registro SOLACI, mostrando a incorporação progressiva das recomendações das diretrizes nesse vasto cenário da vida real e a consequente melhora dos desfechos cardíacos hospitalares. Nau et al. apresentam porcentual de uso, perfil de pacientes e resultados clínicos da trombectomia manual pré-angioplastia primária do Registro Multicêntrico Argentino de Infarto Agudo do Miocárdio com Supradesnivelamento do Segmento ST. Recebemos também de Pedra et al. a interessante experiência inicial multicêntrica na América do Sul do fechamento perventricular de comunicação interventricular muscular com dispositivos Amplatzer via toracotomia mediana.

No cenário dos métodos de imagem, Falcão et al. trazem uma comparação de dois métodos de última geração, na avaliação da placa aterosclerótica coronária. Avaliaram a associação entre a densidade da parede arterial à tomografia computadorizada com múltiplas colunas de detectores e a composição da placa ao ultrassom intravascular com técnica de histologia virtual. Ibraim Pinto, do Instituto Dante Pazzanese de Cardiologia (São Paulo, SP), analisa em seu editorial os resultados de literatura relativos à avaliação das artérias coronárias com as diversas gerações de tomógrafos, admite algumas limitações atuais do método, e mostra-se confiante no futuro próximo, quando essa metodologia possibilitará a monitorização nãoinvasiva da doença arterial coronária.

Por fim, Centemero et al. analisam em profundidade os ainda hoje tão debatidos resultados da revascularização miocárdica em diabéticos. Trata-se de completa revisão, realizada em conjunto com Valentin 
Fuster e Michael Farkouh, do Mount Sinai School of Medicine (New York, Estados Unidos), em que expõem os resultados dos estudos mais significativos do tema, incluindo os recentes BARI-2D e SYNTAX. Trata- se de leitura obrigatória para aqueles que desejam se atualizar no tema. Aproveitem!

Áurea J. Chaves Editora 